The scale is a fundamental concept in geographical analysis, and this book addressed the importance of scale in remote sensing (or broadly GIScience) from different perspectives. This well-organized book includes four themes (13 Chapters), namely scale/multi-scaling issues, physical scale, human scale, and social scale. The first chapter provides a succinct review of the basic concepts of space, time, and space-time in GIScience. It highlights the concern of scale on the past, present, and future of GIScience research, which lays a solid foundation for subsequent chapters. The second chapter introduces the idea of complexity science to a broader audience in layman's term. It covers the fundamental topics of systems (e.g., complex systems, self-organization), framing practice, and the well-known modifiable areal unit problem with various examples in geographical analysis. Chapter 3 starts from a physics perspective to examine issues related to complexity in remotely sensed data. Chapter 4 introduces multiscale modeling and data fusion techniques, and Chapter 5 highlights the importance of error and accuracy assessment in data fusion.

The second theme focuses on the physical scale in relation to natural resource management. Chapter 6 demonstrates a web-based system of fire monitoring and mapping for wildfire suppression planning assistance and postfire damage assessment. Chapter 7 focuses on complexity and scale issues in geomorphology and mountain dynamics. Chapter 8 discusses the problem of scale related to on-demand web mapping services. The third theme is related to human scale in a sense of urban settings. Chapter 9 applies spectral mixing models to analyze urban areas in multiple scales. Chapter 10 integrates LiDAR and high-resolution satellite imagery to identify urban road infrastructure with object-based image analysis technique. The last theme examines the scale of remote sensing data for social consequences including public health. Chapter 11 illustrates how climate and environmental information derived from remote sensing contributes to fighting vector-borne diseases. Chapter 12 describes and discusses the scale of transmission, surveillance, and the control efforts of different infectious diseases. Finally, the last chapter (Chapter 13) provides a meta-analysis from peer-reviewed articles that integrate NASA Socioeconomic Data and Applications Center (SEDAC) products and remote sensing data.

Despite the fact that the first theme is more theory-oriented, the following chapters ultimately show how multiple scale issues combined with the application of geospatial technologies in natural resources, urban studies, and public health. It is worth mentioning that over 20 years ago, a book entitled "Scale in Remote Sensing and GIS" was edited by Drs. Dale Quattrochi and Michael Goodchild. This

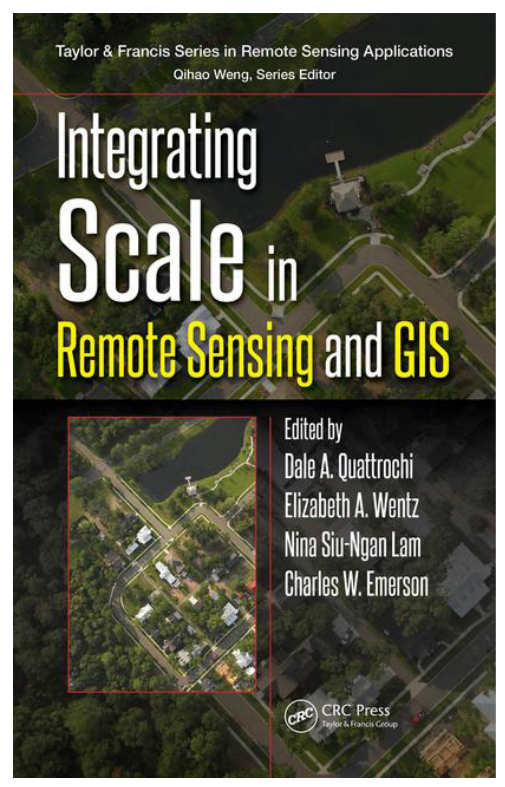

\section{Integrating Scale in Remote Sensing and GIS}

Edited by Dale A. Quattrochi, Elizabeth Wentz, Nina Siu-Ngan Lam, and Charles W. Emerson January 19, 2017, by CRC Press. Hardback: \$160.

Reference - 402 Pages - 134 B/W Illustrations

ISBN 9781482218268

Review by Mingshu Wang, Assistant Professor, Faculty of Geo-Information Science and Earth Observation (ITC), University of Twente, Enschede, Netherlands.

book can be regarded as a continuation of the importance of scale as a foundation for geographical analysis, which indeed has many implications on GIScience applications. Nevertheless, the theories and methods of scale in GIScience still need further exploration.

Photogrammetric Engineering \& Remote Sensing Vol. 84, No. 11, November 2018, pp. 673. $0099-1112 / 18 / 673$

(C) 2018 American Society for Photogrammetry and Remote Sensing doi: 10.14358/PERS.84.11.673 